\title{
Improving the Efficiency of a Photovoltaic Module by Fault Diagnosing, Cleaning \& Dual Axis Solar Tracking Using Internet of Things ( IOT)
}

\author{
Seema Srinivas ${ }^{1,}$ Dr.D.G.Anand ${ }^{2}$, Dr. H.G. Chandrakanth ${ }^{3}$ \\ \{1 ${ }^{1}$ seemasrinivas01@gmail.com, ${ }^{2}$ dga.ece.rgit@gmail.com, ${ }^{3}$ ckgowda@ hotmail.com $\}$ \\ ${ }^{1}$ Research Scholar, Global Academy of Technology, Bangalore \\ ${ }^{2}$ Professor \& Rector-Research, Rajiv Gandhi Institute of Technology, Bangalore, \\ ${ }^{3}$ Principal, Sambram Institute of Technology, Bangalore, Karnataka, \\ Visvesvaraya Technological University, Belagavi, Karnataka
}

\begin{abstract}
A Photovoltaic array monitoring system with wireless sensors are integrated to the PV modules and transfer the status statistics to monitoring field. These sensors are employed for fault detection and observe misbehavior of the cells. In this work we are focusing on voltage, current, irradiance and temperature of individual photovoltaic array and its parameters are studied along with transfer of data's to the controlling unit. The lab view and aurdino boards are used for the development of storing and displaying along with controlling data received from wireless sensor. These received information are used to study the array conditions including fault finding, shades on the panel, loading.
\end{abstract}

Keywords: Aurdino board, Stepper motor, dual axis tracker, PV Module, Self-Cleaning

\section{Introduction}

In the present world, electricity has become a part of our basic need in our life. The energy consumption is rising as our population is increasing while energy production is reducing. The failure of energy has significant socioeconomic implication on consumers. In order to balance the shortage of electrical energy, it is diverted look upon other media to generate clean energy. Due to the pollution and environmental issues related to using conventional fuels and limitations in the sources, renewable energy systems are recommended in various applications for the sake of providing clean energy. In the recent study, it results in reducing the carbon dioxide emission.

Solar Photovoltaic (PV) is used as renewable energy generator to facilitate consumers. This solar photovoltaic cells convert's freely available, unlimited sunlight to electrical energy without pollutants like corbondioxide. This PV generation has been integrated to power grids to produce required quantum of energy. But it has the drawback of occupying larger area for the installations and unpredictable weather conditions. So, it is observed to generate more reliable, effective energy with minimal cost of producing single is very low.

The energy system must be reliable i.e. Secured System with system adequacy. The Photovoltaic Cells are composed of many chemical and electronics components which are mainly depend on the temperature, energy loss, larger space, dust free zone, longer life span of solar panels etc.

These PV cells don't have any moving parts for generating power, but still it faces the failure in interconnections of panels, invertors, batteries \& conditioning components and it's operating and maintenance cost is very nominal. Most of these issues must be resolved at the earliest since it leads to the loss in the production of solar energy .In order to overcome these issues and for the improvement of power generation, PV monitoring and methods are proposed. [1-5], In general, these photovoltaic arrays which are installed at remote places are monitored manually which normally consumes more time and also prone to errors .Thus Photovoltaic array monitoring requires automation for data acquisition, storing and also for controlling. This set of monitoring arrangement and Healthy PV system arrays, significantly improves the overall production of power with efficiency of each panel may be achieved. [6]

\section{Role of Solar Energy}

The renewable energy is playing vital role in the present scenario as, importing fuels stimulating the Indian economy. The initial investment for the installation of the equipment will be very high. But the energy falling on the earth from the sun is good enough throughout the year to meet the need \& demand of the growing population 
\& industry. Our country easily utilize the solar power. The second advantage of using solar power is it generates employment to the youth of this great nation. Inview of the same, this proposed solar powered energy integrated with the recent development in IOT which is used as a caretaker of the instruments, to improve the permanence of equipment and production to its best.

\section{Need for PV Monitoring System and Performance Analysis}

The main objective of any PV system is the monitoring section used to study the performance of the PV system regularly. The proposed monitoring used employ Internet of Things sensors to monitor and study its performance of PV plants under Test.

In the field of solar PV systems, sometimes more difficult to locate the fault so effectively, which may leads to whole system failure. Due this reason, in the PV arrays, fire hazards have been reported because of overheating of solar panels or due to the mismatch of the installed solar panels

\subsection{Overview of PV system faults.}

These are subject to a variety of faults, which includes partial shading (PS)conditions which intern have impact on the output power of the PV system, the maximum Power point tracking (MPPT) units, PV hot spots, and PV micro cracks.

\subsection{Factors influencing the performance of PV modules}

- PV micro cracks : The PV micro cracks, This PV micro cracks occurs in the PV panels due to environmental conditions, manufacturing defects, variations in temperature and humidity. These micro cracks in the module reduces the efficiency by $8 \%$ to $9 \%$.

- Hot Spots : This is one of the important factor, which reduces the output power of PV modules. Therefore, hotspots detection technique is of very much importance in these setups. This power loss could be reduced by taking corrective mitigating techniques to reduce its impact on the performance of PV panels.

- $\quad$ Faulty PV Modules : This refers to the PV module which is totally disconnected from that PV arrays. In other words, a faulty panel refers to a short circuited PV arrays.

\section{Need for Internet of Things (IOT) in Solar PV Monitoring}

A low cost IOT system consisting of wireless sensor network deployed with solar PV monitor system plays an important in collecting PV solar array information. These received data's through Internet at remote place helps in studying the health condition of solar array, machines used in installation with electrical fixtures, failure status and regular maintenance along with quality and quantity of solar efficiency.

\section{Scope of the Work}

This research work focuses on reliability of energy system with renewable power generation. To investigate the effects of various key factors like temperature, climatic condition, pollution and other parameters related with energy generation in terms of these elements impact on the system reliability. The evaluation of PV sources and its effect on other related parameters issues which effects on overall performance of the system.

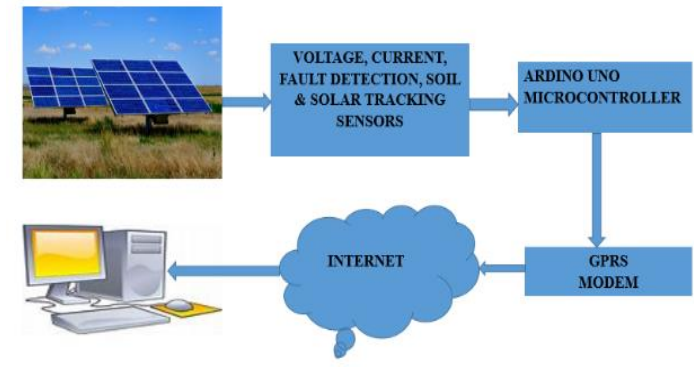

Fig 1.Concept of smart PV monitoring system 


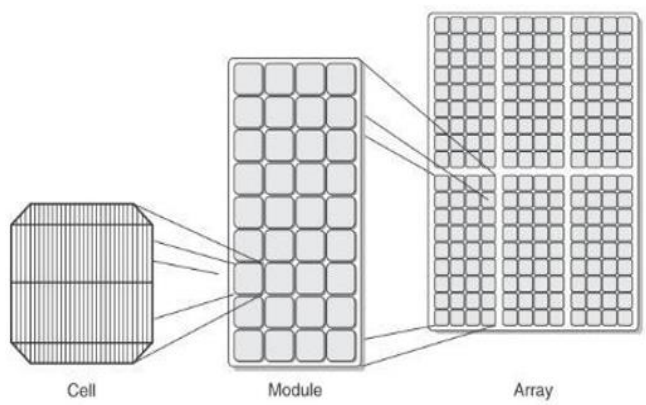

Fig 2. Solar Cell, Module \& Array

\section{Background Theory}

The sun tracking is a device that rotate in up \& down along with right to left direction. This arrangement can be PV panels, lenses, mechanical arrangements for fixing / tracking system. This trackers role is to trace the sun and also to check the angle of incidence, so as to have the best radiation falls on the PV panels by taking care of temperature coefficient. The direct beam falls on the panel delivers $90 \%$ of the energy. As the majority of the energy is collected in this angle, the focusing is to have same energy as long as sun is in clear sky. Using the ingrated dual axis sun tracker is employed to track the solar system which has azimuth $\&$ altitude tracing.

\section{Literature Survey}

The single axis tracker for tracking the sun along the horizontal direction and other vertically was presented. The energy was focused using lens \& mirror into narrow beam, then this energy was converted into electrical current using photoelectric phenomenon. The output increased from $26 \%$ to $38 \%$ compared to standard fixed mode of operation on normal days [7].

Normally, PV system will have sensing device like current sensors and voltage sensors with inverters. Sun tracking system was developed to work effectively and also to receive the related parameters from sensors. This developed two -axis sun tracking has been installed with microcontrollers based electronic controlling arrangement. This tracker based arrangement delivered best performed by $26 \%$ on normal days and $21 \%$ on cloudy day [8]

A PV array panel tracking system was developed to track sun. This arrangement had the advantage of auto tracking system at random time to diagnose and face towards sun. After $180^{\circ}$ rotation, aligned to receive quantum of solar energy, it used to go for rest mode. It had the advantage of stepper motor for the rotation /tracking for ensuring very high vitality improvement. [9]

Divya, proposed a system to overcome the drawback of reduction in producing solar energy due to dust \& pollution [10]. The system designed and developed an automated system for sensing and clearing at regular interval. This was developed using AurdinoUNO. This work had the advantage of increase in energy production over non cleaning PV array.

In Ref. [11], designed and developed an integrated system which includes tracking sun on both azimuth axis and altitude axis along with automated cleaning technology to achieve optimum efficiency of the system. They developed an experimental setup to monitor the system using various sensors. The authors observed the improved efficiency of the solar energy compared to previous arrangements.

\section{Problem Definition}

The Output power of Photovoltaic (PV) panel directly proportional to the irradiation \& inversely to the temperature. Hence if temperature during sunny days improves, current improves with decreasing in voltage \& intern net power also decreases. Secondly, majority of PV panels are installed in outdoor which definitely in harsh, remote environment, which affects by system fault. This may be due to the shading, overheating of system, electrical system - cabling or invertor or due to aging factor. So, monitoring of all parameters for conditioning of PV panels \& also fault diagnosis of PV cells and related arrangement are very much essential for better reception of PV system and better production of solar energy. 


\section{Hardware Implementation of the Tracker}

The conversion efficiency of a solar panel is directly proportional to the amount of direct solar irradiance that is absorbed. Irradiance is the amount of solar radiation that strikes the surface of a solar cell or panel and it is expressed in $\mathrm{kW} / \mathrm{m}^{2}$. The irradiance multiply by time is a measure of solar insolation.

The peak sun hours is the number of hours per day when the solar insolation $=1 \mathrm{kw} / \mathrm{m}^{2}$.A solar tracking system is an arrangement made or adjust the direction and height of the solar PV panels to face towards the sun. The sun's position changes with day and seasons as the sun moves across the globe. These solar panels absorbs energy from the sun better in perpendicular direction to the sun. Hence the solar tracking system is an important activity in power generation and illustrated in fig 3. The energy genera rated from the fixed solar array will improved by employing tracking system. Various factors are to be studied includes the azimuth angle of installation, climatic condition, and cost of installation \& maintenance of the tracking arrangements among others.

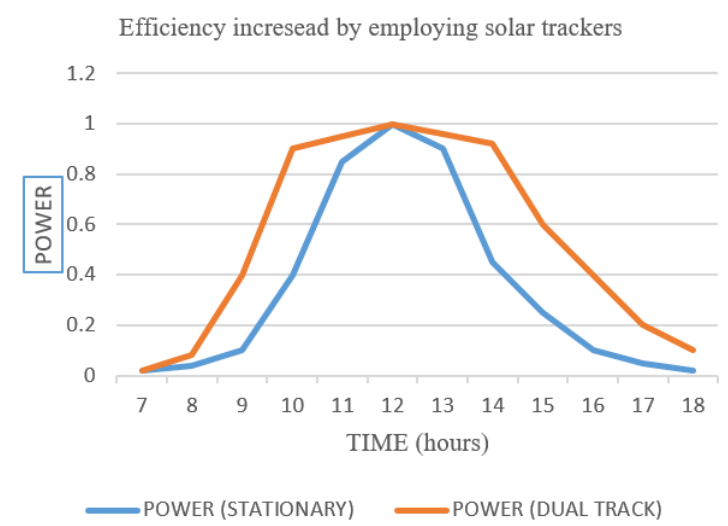

Fig.3 . Efficiency increased by employing solar trackers

In this paper, to monitor the real-time data like electrical PV array voltage, current, solar irradiance and temperature of the panels are collection, we have developed prototype model at our research Centre. This prototype could be utilized to find out the PV panels health monitoring by integrating additional diagnosing electrical equipment and connections along with inverter board.

\section{Working of Proposed System}

This work presents a solar array plant based on Internet of Things [IOT] with Aurdino Uno board, sensing arrangement for gathering the data along with computing facilities. Here, we are employing many types of sensor nodes for sensing various parameters such as soil, humidity, temperature and voltage and current. These parameters are very much required to understand the performance of the solar array, stepper motors, inverters etc. for diagnosis of all related components performance and electrical system along with self-cleaning arrangements to clear \& clean the PV module at regular intervals. This fault finding or health checkup of solar panels and related devices through IOT by data acquisition \& controlling helps to produce more energy and also to avoid breakdown by taking preventive measures.

The user can check \& monitor the environmental parameters stored in cloud, thereby the receiver can avail all related values through wireless sensor nodes $\&$ internet wherever $\&$ whenever it is required.

\section{Efficiency Calculation}

\section{A. For Clean Panel}

For calculation of solar panel efficiency,

Efficiency $=\mathrm{P}_{\text {lax }} /($ Radiation Intensity $*$ Area $)$

$\mathrm{P}_{\text {lax }}=\mathrm{A}_{\max } * \mathrm{I}_{\mathrm{MAX}}$

Area of Panel $=$ Length $*$ Breadth

Thus, Efficiency $=\mathrm{P}_{\text {lax }} /$ (Area of the Panel $*$ Radiation Intensity) $=\mathrm{X}_{\mathrm{CLEAN}}$ 


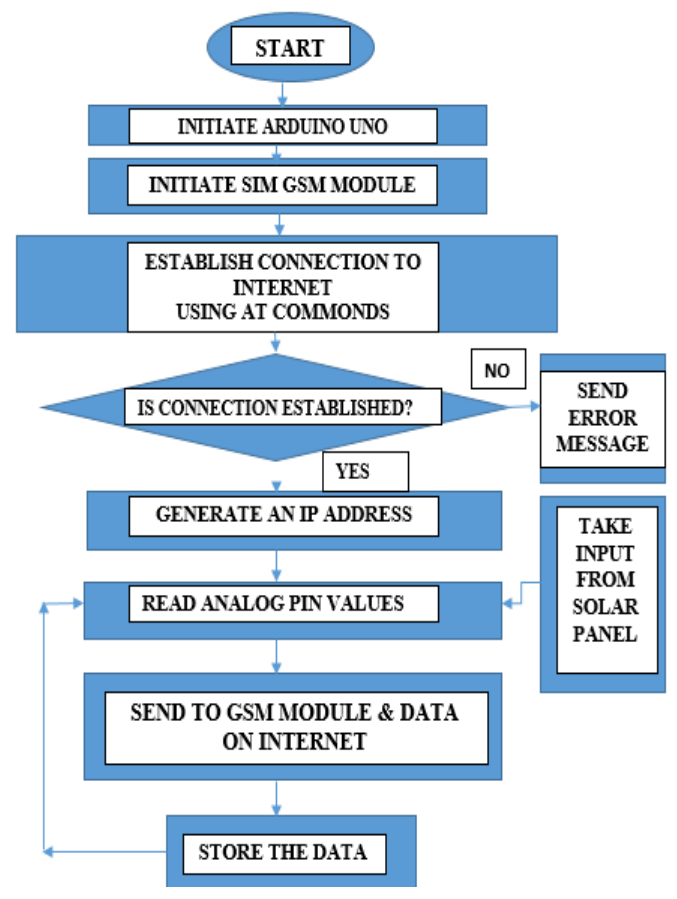

Fig 4. Flow Chart

\section{B. For Unclean Panel}

For calculation of solar panel efficiency,

Efficiency $=\mathrm{P}_{\max } /($ Radiation Intensity $*$ Area $)$

$\mathrm{P}_{\max }=\mathrm{V}_{\max } * \mathrm{I}_{\max }$

Area of Panel $=$ Length $*$ Breadth

Thus, Efficiency $=\mathrm{P}_{\max } /($ Area of the Panel $*$ Radiation Intensity $)=\mathrm{Y}_{\text {UNCLEAN }}$

\section{Percent Decrease}

The formula used to calculate Percent Decrease in efficiency is,

Percent Decrease $=[($ Efficiency of Clean Panel - Efficiency of Unclean Panel $) /($ Efficiency of Clean Panel $)] *$ 100

Thus, Percent Decrease $=\left[\left(\mathrm{X}_{\mathrm{CLEAN}}-\mathrm{Y}_{\mathrm{UNCLEAN}}\right) / \mathrm{X}_{\mathrm{CLEAN}}\right] * 100=\mathrm{Z} \%$

Above calculations proves that due to the continuous accumulation of dust on solar panel surface area, the efficiency of panel decreases by Z \%.

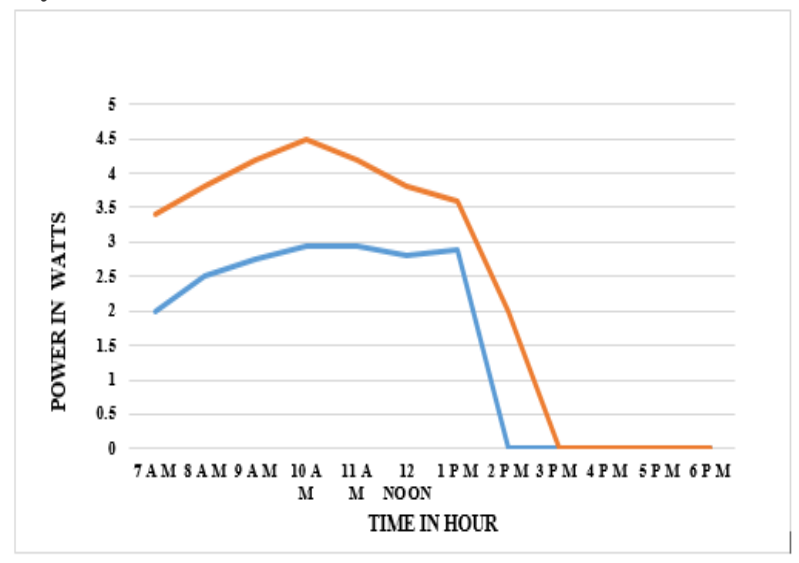

Fig 5. Power comparison between fixed \& tracking solar system on cloudy day 


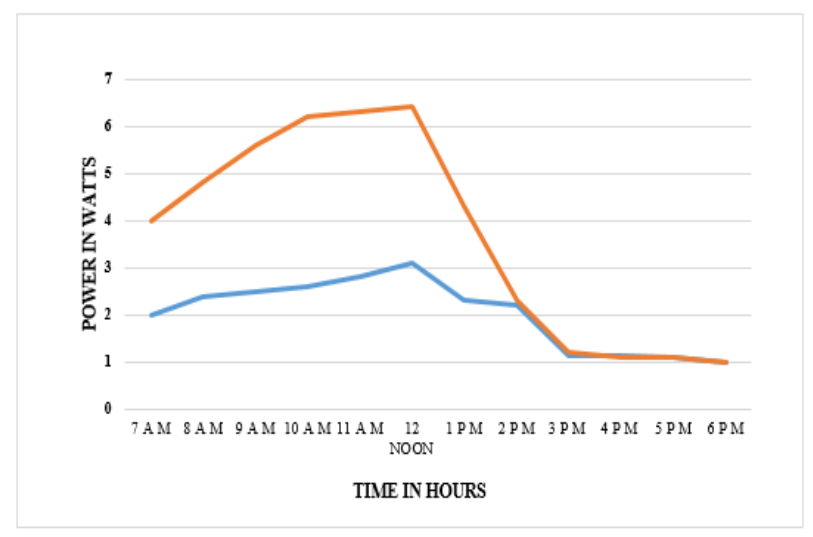

Fig 6. Power comparison between fixed \& tracking solar system on summer day

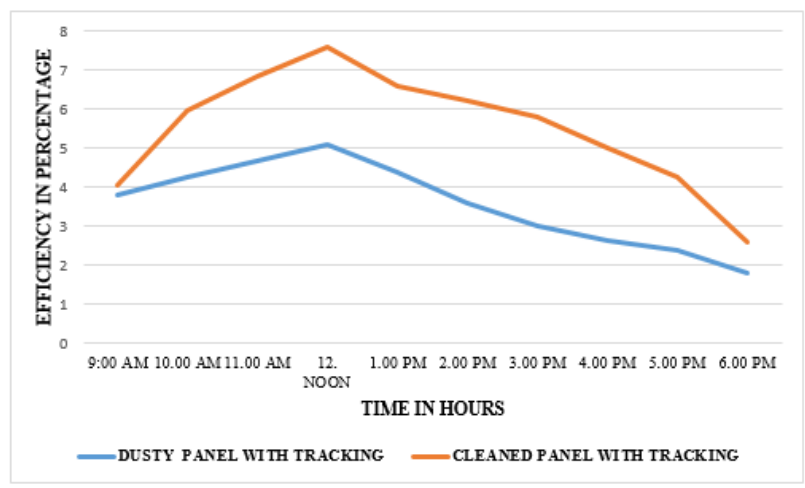

Fig 7. Comparison between dusty panel with tracking \& clean panel

\section{Results}

We have recorded the values of voltage and current at regular intervals both on sunny day \& also on cloudy day. This has been represented in the figures $5,6 \& 7$ which proves that the efficiency can be improved by cleaning of panels with dual tracking arrangements. The power efficiency on sunny day yielded the best result. We have also recorded and compared readings between uncleansed dusty PV panel \& automated wiper cleaned PV panel. The comparative study between shows that the cleaned PV panel produced better efficiency.

\section{Conclusion}

This work has many advantages since it is the integration of cleaning, fault diagnosing along with solar tracking. We have shown that this arrangement will be working throughout the year $\&$ in all climatic conditions and also deliver best result. Now at present good number of researchers are working on these issues to monitoring the PV array using sensor network with different chemical structures and also monitoring techniques to avoid failures by supervising the system for the better energy efficiency. Hence main challenge is will be to derive a mechanism for excellent diagnosing system that can detect and diagnose all the PV system failure along with accurate results. Hence the proposed arrangement delivers the improvement in energy status.

\section{References}

[1] www.bluesystem.ca. : Laurent Mingo, "A Cost-effective Solution for Photovoltaic Panels Performance Monitoring Using NI Lab VIEW", Blue System Integration Ltd

[2] International journal of hydrogen energy : Dimitris Ipsakisa, Spyros Voutetakisa, Panos Seferlisa, Fotis Stergiopoulosa, \& Costas Elmasidesb, "Power management strategies for a stand-alone power system using renewable energy sources and hydrogen storage", vol 34, pp. 7081-7095, 2009.

[3] Proc. World Renewable Energy Congress : M.B. Zahran, \& O.A. Mahgoub, "Photovoltaic Battery (PVB) Stand-alone System Control Signals Estimation", VI, 2000, pp. 2119-2122. 
[4] ISESCO Science and Technology Vision : Mohammad Zakir Hossain, \& A.K.M. Sadrul Islam, "PV wind Hybrid System Modelling for Remote Rural Application", vol. 3(4), November 2007, 59-64.

[5] Electronics Kashia Jived,Harpoon Asha,Raj veer Singh,S.M. Shall Husain and Tasha Slim Stun, "Design and Performance Analysis of a Stand-alone PV System with Hybrid Energy Storage for Rural India", 2019, 8(9), 952;

[6] Computer Geoscience, P.S.Dahl, A PC and Lotus-based data acquisition/reduction system for an ICP spectrometer, 16(7), 2000, 881-892.

[7] International Journal of Scientific \& EngineeringResearch : K.S.Madhu, B.R.Wadekar, Finavivya, Chiragkumar.V, Gagan.T.M,“Intelligent Two Axis Solar Tracking System with Mechanical Application” vol. 3, 2229-5518, 2012.

[8] International Journal of Advances in Science Engineering and Technology: Adarsh S., Abhishek Anand, Jayant Singla "Increasing the efficiency of a PV system using dual axis solar tracking " ISSN: 2321-9009 Volume- 3, Issue-2, April-2015

[9] International Journal of Technical Innovation in Modern Engineering \& Science (IJTIMES) : S.Sowmya, Dr.M.Yohan "Sun tracking solar panel by coupled with a stepper motor",e-ISSN: 2455-2585 Volume 4, Issue 09, September2018

[10] International Journal of Advance Research, Ideas and Innovations in Technology: R. Divya, K.Sheeba, J.Gayathri dhevi \& S.Sandhiya "Automatic Cleaning of Solar Panel with Maximum Power Tracking by using Aurdino", Volume 4, Issue 2,PP 324-328,2019

[11] International Journal of Advanced Research in Electrical, Electronics and Instrumentation Engineering: Sujata Shivashimpiger, Sri Lakshmi C.S. "A Combined approach Towards the Efficiency Improvement of Solar Photovoltaic Panel "Vol. 6, Issue 6, June 2017, pp. 4903-490. 